\title{
A mixed design reveals that glucose moieties facilitate extinction of a conditioned taste aversion in rats
}

\author{
MICHAEL E. SMITH, RALPH NORGREN, and PATRICIA SUE GRIGSON \\ Pennsylvania State University College of Medicine, Hershey, Pennsylvania
}

\begin{abstract}
Separate groups of water-deprived rats had four trials with 15-min access to $0.0073 \mathrm{M}$ saccharin, $0.3 \mathrm{M}$ alanine, $0.3 \mathrm{M}$ glucose, $0.1 \mathrm{M}$ maltose, $0.3 \mathrm{M}$ fructose, $0.06 \mathrm{M}$ sucrose, or $0.03 \mathrm{M}$ Polycose. Trials 1-3 were followed by injections of either $0.15 \mathrm{M} \mathrm{LiCl}(1.33 \mathrm{ml} / 100 \mathrm{~g}$ b.w., i.p.) or saline; Trial 4 (Test) was CS only. Extinction included either 48-h access to water alone or to the appropriate CS, both followed by a 24-h, two-bottle choice of CS and water. This 3-day cycle was repeated five to six times. All rats acquired comparable conditioned taste aversions (CTAs), but extinction rates varied with the test and the taste CS. No CTA extinguished during the two-bottle choices following 2 water days. During one-bottle CS exposure, all CTAs extinguished, but the aversion continued longer in the probe twobottle tests. Intake of glucose moieties recovered rapidly, often in two cycles; the other CSs took four to six cycles. Thus, CTA extinction varies with the nature of the taste CS.
\end{abstract}

A conditioned taste aversion (CTA) occurs when intake of a gustatory conditioned stimulus (CS) is reduced following pairing with an aversive agent such as lithium chloride (LiCl) or X-radiation (Garcia \& Koelling, 1966; Spector, Smith, \& Hollander, 1986). In comparison with many other learning phenomena, CTA learning is robust and has a number of unusual characteristics. For instance, the association between the gustatory CS and the aversive unconditioned stimulus (US) can occur following a single CS-US pairing. The CTA can occur with a long CS-US interval (e.g., 24 h). At least in rodents, the animals appear to be prepared to associate a gustatory CS with gastrointestinal malaise (Garcia \& Koelling, 1966; Revusky \& Garcia, 1970; Seligman, 1970). Considerable experimental attention has been given to these characteristics, all of which are related to the acquisition of a CTA (see Klosterhalfen \& Klosterhalfen, 1985). Since its discovery and initial characterization, extensive analysis has revealed much about the physiological, biochemical, and neural mechanisms mediating the acquisition of a CTA (Berman \& Dudai, 2001; Gutiérrez, HernándezEcheagaray, Ramírez-Amaya, \& Bermúdez-Rattoni, 1999; Norgren \& Grigson, 1996; Reilly, 1998, 1999; Spector, 1995).

This research was supported by National Institutes of Health Grants DC-00240, DA-09815, DA-12473, and DK-09926. A portion of the data described in this study was presented at the 1993 Eastern Psychological Association meeting, Washington, DC. We thank Polina Lyuboslavski and Sarah Wolpert for their contributions to an earlier version of the manuscript. Correspondence concerning this article should be addressed to M. E. Smith, Department of Neural and Behavioral Sciences, College of Medicine, Pennsylvania State University, Hershey, PA17033 (e-mail: mes32@psu.edu).
In contrast, our understanding of the mechanisms mediating the extinction of a CTA is limited. Extinction is defined as a decrease in the expression of the conditioned response following repeated, unreinforced presentations of the CS. Although this definition seems relatively straightforward, there is disagreement about how best to produce and assess extinction of a CTA. Complete extinction of a CTA has been reported when using either one-bottle or two-bottle tests (Nolan et al., 1997). There are benefits and limitations with either method. For example, some evidence suggests that two-bottle tests (i.e., simultaneous access to the CS and water) are more sensitive than one-bottle tests for detecting a more persistent and graded CTA (Grote \& Brown, 1971). Intake of the CS during a two-bottle test, however, may remain very low because the rat need not sample a CS delivered in the presence of water. On the other hand, in a one-bottle test, CS intake can be deceivingly high because the CS serves as the only source of fluid.

In an effort to maximize the positive features of both procedures and to reduce the impact of the negative features, the present study evaluated extinction of a CTA using a combination of each. In this new paradigm, referred to as the mixed extinction design, rats trained on a CTA were given forced exposure to the CS during a onebottle extinction test for $48 \mathrm{~h}$ and then were probed for the expression of the CTA using a 24-h two-bottle choice test (CS vs. water). This 3-day cycle was repeated until intake of the CS matched that of water (i.e., for five to six cycles). It was hypothesized that, although the nature of the CS appears not to affect the acquisition of a CTA, it might affect the rate of extinction. Indeed, features of the CS have been found to affect the rate of extinction in other paradigms (Taylor \& Boakes, 2002). Thus, the ac- 


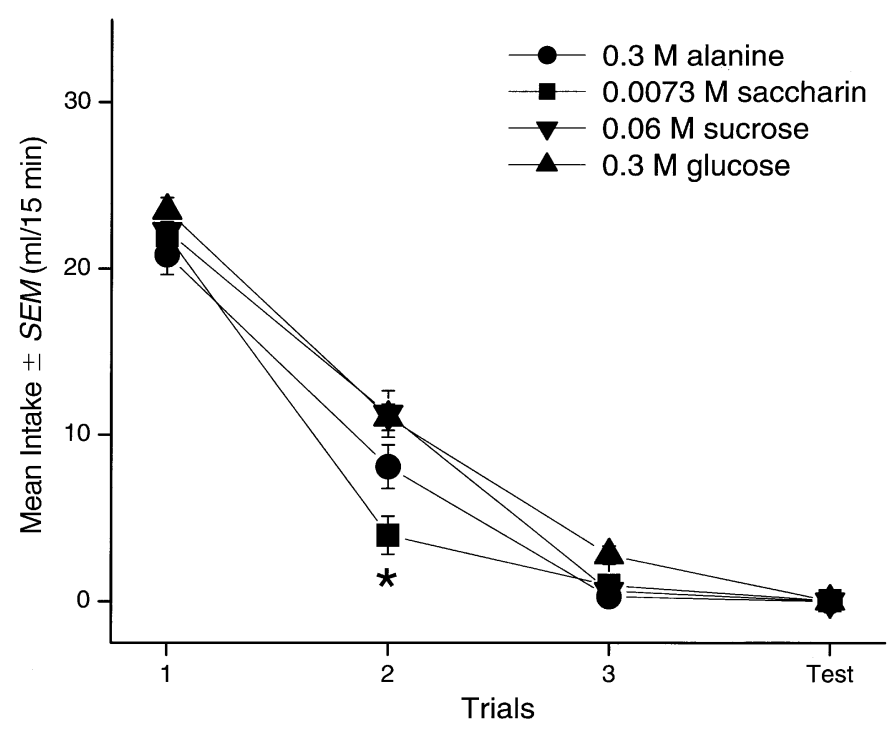

Figure 1. Mean ( $\pm S E M)$ intake of CS solutions during the three 15min conditioned taste aversion (CTA) acquisition trials and one 15-min CTA test trial in Experiment 1. The asterisk indicates a difference in intake on Trial 2 between saccharin and the three other CSs $(p<.05)$.

quisition and extinction of an LiCl-induced CTA were examined across two experiments using a range of CSs including monosaccharides (glucose, fructose), disaccharides (sucrose, maltose), and a polysaccharide (Polycose), as well as saccharin and an amino acid (alanine). When presented with water, all of these compounds are preferred by rats and, except for Polycose, all taste sweet to humans.

\section{EXPERIMENT 1}

Experiment 1 examined the role of the gustatory CS in the acquisition and extinction of a CTA by pairing saccharin, sucrose, glucose, or the amino acid alanine with $\mathrm{LiCl}$. The rate of extinction of the CTA was then examined in a group of rats tested using the mixed extinction design and in a group of rats tested using the standard two-bottle procedure only. Using this approach, it was possible to measure when using only a two-bottle test whether rats would fail to sample the CS, and when using the mixed design, whether rats would express a CTA during the two-bottle probe test that was not evident during the one-bottle forced exposure.

\section{Method}

\section{Subjects}

The subjects were 59 male, Sprague Dawley rats (Charles River Laboratories, Wilmington, MA) weighing approximately $400 \mathrm{~g}$ at the start of the experiment. They were individually housed in suspended stainless steel cages in a temperature-controlled colony room maintained on a 12:12-h light:dark cycle. The rats were given free access to standard laboratory chow (Rodent Laboratory Chow 5001, Ralston-Purina, St. Louis) but were restricted to $15-\mathrm{min}$ access to distilled water daily.

\section{Apparatus}

The taste stimuli were prepared daily using distilled, deionized water. The stimuli included $0.0073 \mathrm{M}(0.15 \% \mathrm{w} / \mathrm{v})$ saccharin, $0.3 \mathrm{M}$ $(2.67 \% \mathrm{w} / \mathrm{v})$ alanine, $0.3 \mathrm{M}(5.41 \% \mathrm{w} / \mathrm{v})$ glucose (Sigma Chemical Co., St. Louis, MO), and $0.06 \mathrm{M}(2.0 \% \mathrm{w} / \mathrm{v})$ sucrose (Fisher Scientific, Fair Lawn, NJ). These concentrations were selected because they elicited a similar amount of intake in a 30-min one-bottle test (Sclafani \& Clyne, 1987); that is, they were matched on acceptance. All solutions were prepared $24 \mathrm{~h}$ in advance and were presented at room temperature in inverted Nalgene graduated cylinders with silicone stoppers and stainless steel spouts. Intake was recorded to the nearest $0.5 \mathrm{ml}$.

\section{Procedure}

Fluid deprivation. All rats were adapted to a water deprivation regimen in which they were given 15 -min access to water each morning (at 0900). Once morning intake stabilized (approximately 2 weeks), the subjects were matched on the basis of their 15-min water intake (averaged over the final 2 days of baseline) and assigned to one of the following CS conditions: saccharin $(n=12)$, sucrose $(n=12)$, glucose $(n=23)$, or alanine $(n=12)$. The sample size was larger for the glucose group because data from an unpublished experiment suggested that the development of an LiClinduced CTA may be delayed when using glucose.

Conditioning. During the conditioning phase, all rats were given 15-min access to the appropriate CS and, after an additional 15-min interval, were injected intraperitoneally (i.p.) with $0.15 \mathrm{M} \mathrm{LiCl}$ $(1.33 \mathrm{ml} / 100 \mathrm{~g}$ b.w.). There were three such CS-US pairings, followed by a single 15 -min test. Two water days ( 15 min access) elapsed between the pairings and the test. Because extinction can be influenced by the strength of the initial aversion (Mikulka \& Klein, 1980), the inclusion of animals in the extinction phase of the experiments required that the rats demonstrate robust CTA learning (i.e., a 75\% reduction in CS intake from Trial 1 to Trial 3). As such, differences in the rate of extinction could not be attributed to differences in acquisition of the CTA. On the basis of this requirement, the data from just 3 rats out of 23 in the glucose group were eliminated from the experiment. 
Extinction. The extinction phase of the experiment began immediately following the final 15-min CTA test. The rats in each CS condition were subdivided into two treatment groups. Group Water received 48-h continuous access to water, followed by 24-h twobottle access to water and the appropriate CS; $n=4$ for each CS subgroup except glucose, where $n=5$. Group CS received 48-h continuous access to the CS that had been used during training, followed by the same 24-h two-bottle test described for Group Water; $n=8$ for each subgroup except glucose, where $n=15$. There was no difference in the rate of CTA acquisition between rats assigned to Group Water or Group CS ( $p>.1)$. Although these tastants are generally preferred to water, extinction of the CTA was operationally defined as equal intake of the CS and water in the twobottle tests. This 3-day cycle was repeated five or six times (until the operational definition of extinction was met), and the left/right position of the solutions was counterbalanced across repeated twobottle tests

\section{Data Analysis}

The data from each of the experiments were analyzed using repeated measures analyses of variance (ANOVAs; Statistica, StatSoft, Tulsa, OK). When appropriate, post hoc tests were conducted using Tukey's honest significant difference tests with an $\alpha$ level set at .05 . The results are shown as the mean \pm standard error of the mean $(S E M)$

\section{Results and Discussion}

\section{Acquisition}

Figure 1 shows intake of the gustatory CSs across three taste- $\mathrm{LiCl}$ pairings and one CS-only test. Post hoc tests of the significant CS $\times$ trial interaction $[F(9,156)=$ $4.02, p<.0001]$ confirmed that, although intake of the gustatory CSs did not differ on Trial $1(p \mathrm{~s}>.05)$, the initial rate of acquisition of the CTA varied as a function of the nature of the CS. Rats in the saccharin-LiCl group exhibited a greater suppression of intake following their initial CS-US pairing (Trial 2) than did rats that received access to either glucose or sucrose $(p s<.05)$. In spite of this difference in the initial rate of acquisition, the magnitude of the LiCl-induced CTA did not differ as a function of CS condition on the third CS-US pairing or the final CS-only test.

\section{Extinction: Group Water}

Figure 2 shows mean $( \pm S E M)$ 24-h intake of water versus CS (sucrose, saccharin, alanine, or glucose) across repeated two-bottle tests of the rats that received water on the intervening one-bottle days. As is evident in the figure, rats that received water during the 48-h onebottle access periods (i.e., the group that did not have forced exposure to the CS) consumed little if any of the CS during repeated two-bottle tests. This conclusion is supported by a significant main effect of stimulus $[F(1,14)=$ $184.4, p<.001]$, showing that intake of water was well above CS intake overall. This pattern was not affected by the nature of the $\operatorname{CS}[F(3,14)=1.18, p>.1]$, nor did it change as a function of training, as demonstrated by a
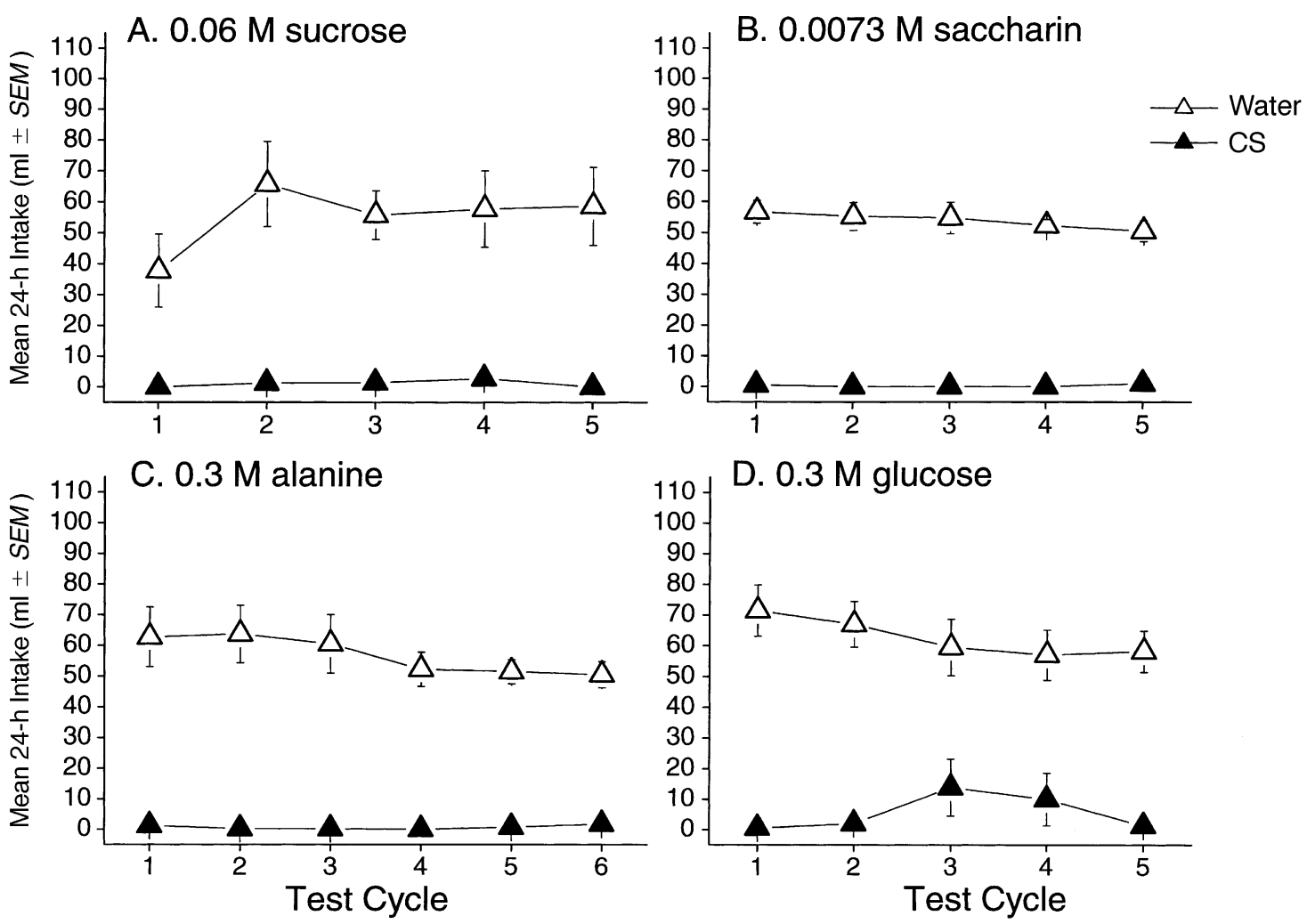

Figure 2. Group Water. Mean ( $($ SEM) intake of water (open symbol) and CS solutions (filled symbol) during the 24-h, two-bottle choice tests in Experiment 1 by the rats that had access to water on the one-bottle days. 
nonsignificant stimulus $\times$ trial interaction $[F(12,56)=$ $1.36, p=.1]$.

\section{Extinction: Group CS}

Figure $3 \mathrm{~A}$ depicts the mean $( \pm S E M)$ intake of water and CS solutions during the two-bottle tests for rats receiving CS on the intervening one-bottle days. Figure 3B compares the mean ( \pm SEM $)$ 24-h CS intake during both the one-bottle and two-bottle access periods. The onebottle intake is the mean 24-h intake during $48 \mathrm{~h}$ of continuous CS access.

CS versus water intake (two-bottle intake). If the rats were presented with only the CS for 2 days, when given a choice on Day 3, they still rejected the CS in favor of water (Figure 3A). On subsequent two-bottle tests, however, they all began to ingest the CS, eventually reaching parity with water intake (Trials 4-6). This observation was confirmed by the significant main effects of CS $[F(3,34)=4.05, p<.05]$, and stimulus $[F(1,34)=$ $20.04, p<.001]$. Post hoc tests on the significant CS $\times$ stimulus interaction $[F(3,34)=35.39, p<.001]$ revealed that intake of glucose was greater than intake of water overall but intake of water was greater than intake of all other gustatory stimuli-that is, saccharin, sucrose, and alanine $(p$ s $<.05)$. Most importantly, the rate of CTA extinction depended upon the nature of the gustatory CS employed, as revealed by post hoc comparisons of the significant three-factor interaction $[F(12,143)=3.87$, $p<.0001]$. Specifically, extinction of the CTA to glucose occurred by the second two-bottle test $(p>.05)$ and extinction of the CTAs to sucrose and saccharin occurred by the fourth test $(p>.05)$. A separate $2 \times 6$

\section{A. CS vs. Water Intake (Two-Bottle)}
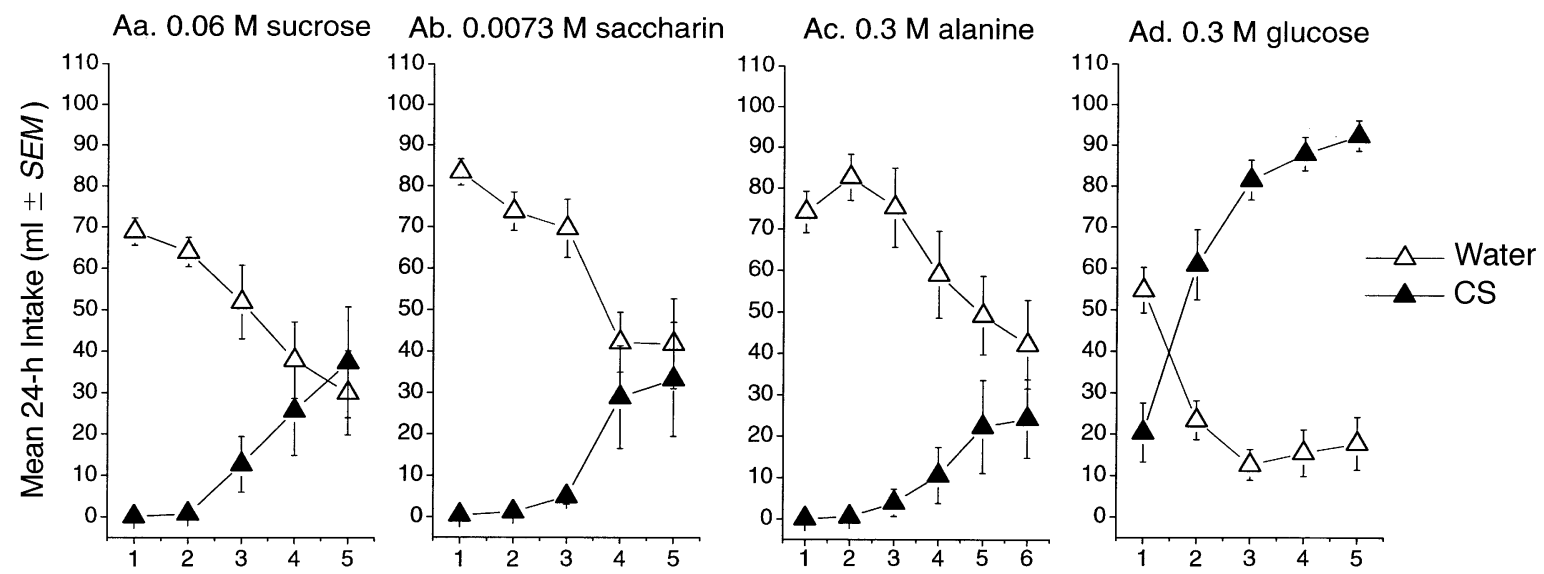

B. CS Intake One-Bottle vs. Two-Bottle
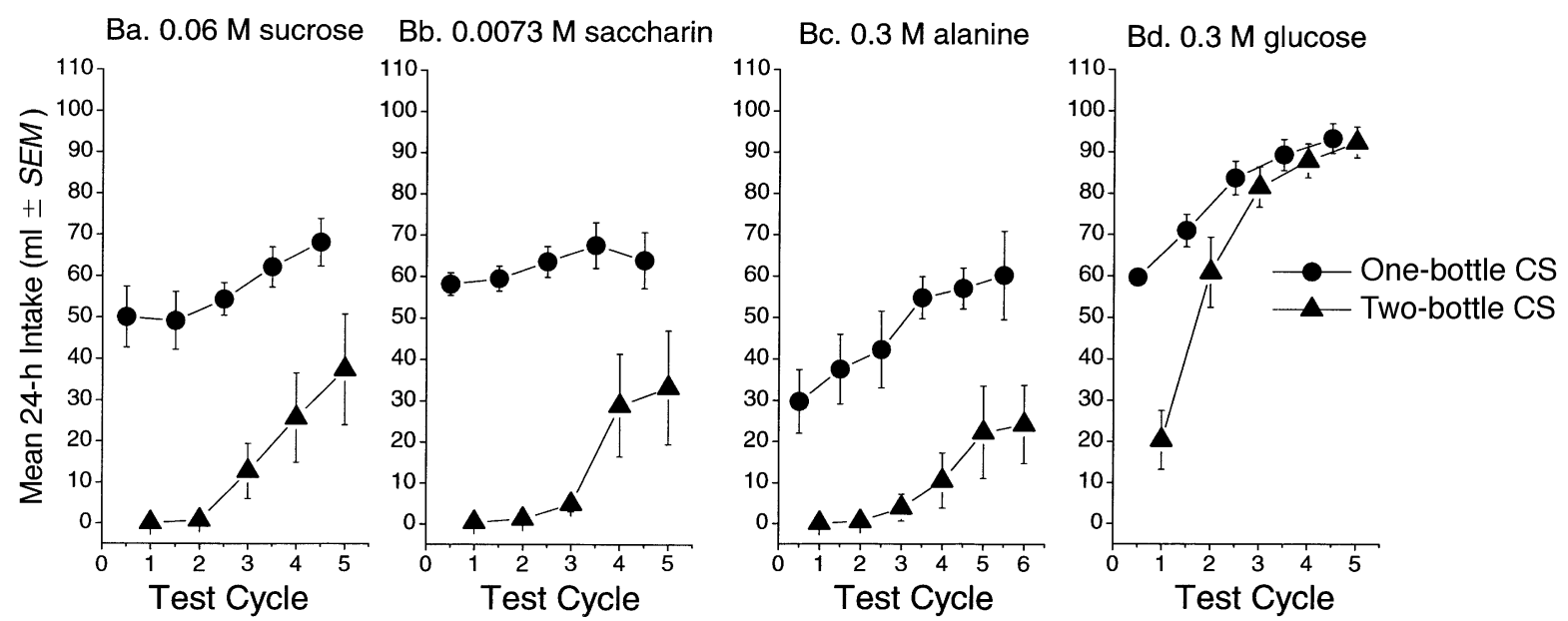

Figure 3. Group CS. The top panels (A) show the mean ( \pm SEM) 24-h intake of water (open triangles) and CS solutions (filled triangles) by Group CS rats during the two-bottle choice tests in Experiment 1 . The bottom panels (B) show the mean ( $\pm S E M)$ 24-h CS intake during both the one-bottle (filled circles) and two-bottle (filled triangles) access periods. The one-bottle intake is the mean 24-h intake during the $48 \mathrm{~h}$ of continuous access to the CS. 
ANOVA on the alanine data confirmed that extinction of the CTA to alanine occurred by the sixth test $(p>.05)$.

CS intake (one-bottle vs. two-bottle). Initially, twobottle CS intake was well below one-bottle levels, regardless of the CS moiety (Figure 3B). By Trial 2, twobottle glucose intake was equivalent to the preceding one-bottle day, as evidenced by the significant $\mathrm{CS} \times$ bottle interaction $[F(3,34)=19.84, p<.001]$. In contrast, post hoc analysis of the significant three-factor interaction $[F(12,143)=3.31, p<.001]$ revealed that even though they increased across trials, two-bottle intakes of sucrose, saccharin, and alanine never approached the one-bottle volumes. That is, intakes of saccharin, sucrose, and alanine were consistently greater during forced exposure (i.e., during the one-bottle test, when a water alternative was not provided; $p \mathrm{~s}<.05$ ).

The results demonstrate the following points: First, at the concentrations selected, the rats consumed similar amounts in $15 \mathrm{~min}$ upon their initial exposure to the four gustatory CSs. Second, using a potent dose of $\mathrm{LiCl}$, acquisition of the CTA was not greatly affected by the nature of the gustatory CS. By Trial 4, which was in extinction, intake of each CS was essentially zero. Due to the floor effect, overtraining cannot be excluded, but the near parallel learning curves (Figure 3) make it unlikely that the final differences between CSs were large. Third, when separated by 2 water days, two-bottle tests alone do not facilitate the extinction of a CTA, because the animals consistently avoid intake of the CS when tested in the presence of a water alternative. Fourth, as with the two-bottle test, exclusive use of the one-bottle test also appears insufficient and may, in fact, yield data that are misleading. Specifically, all rats consumed large quantities of the CSs in the forced choice one-bottle test, despite the fact that they avoided the same CS when subsequently given a choice in the two-bottle test. Forced choice, then, can mask the expression of a CTA. Fifth, the combination of these two procedures in the mixed design is superior to either alone. The one-bottle test favors exposure to the CS, whereas the two-bottle test serves as a probe to test for the expression of the CTA. Thus, neither one-bottle forced CS exposure nor the twobottle probe after $48 \mathrm{~h}$ of water by themselves differentiated between the taste stimuli. When assessed with the two-bottle test in the mixed extinction design, however, the four CSs clearly differed. Extinction (i.e., equal intake of the CS and water) occurred by the second twobottle test for glucose, by the fourth for sucrose and saccharin, and by the sixth for alanine. Finally, intake of saccharin, sucrose, and alanine reached only a point of indifference by the fifth cycle (i.e., the animals consumed $50 \% \mathrm{CS}$ and $50 \%$ water), whereas a $2: 1$ preference was evident for glucose over water by the second cycle and increased to a 9:1 preference by the fifth cycle.

\section{EXPERIMENT 2}

The rapid extinction of the CTA to glucose relative to the other three CSs was unexpected. Because glucose and sucrose are both sugars but had different extinction profiles, this second experiment reevaluated CTA acquisition and extinction using two monosaccharides (glucose and fructose), a glucose dimer (maltose), and a glucose polymer (Polycose) as the CSs. The selected concentrations

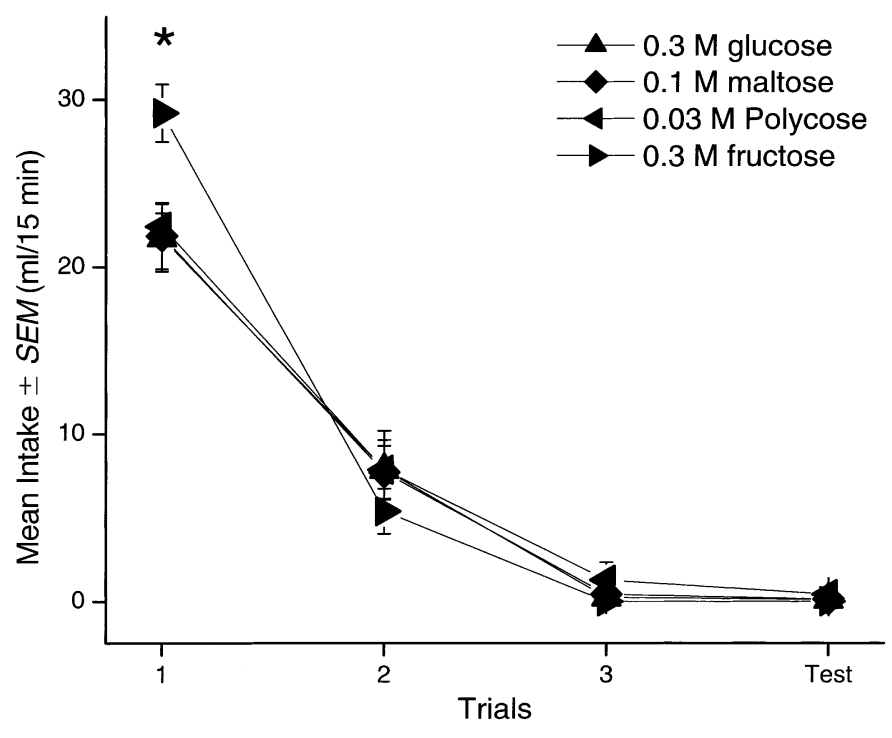

Figure 4. Mean ( $\pm S E M)$ intake of CS solutions during the three 15min CTA acquisition trials and one 15-min CTA test trial in Experiment 2 . The asterisk indicates a significant difference in intake between fructose and the other three CSs on Trial $1(p<.05)$. 
were expected to support equal intake in the first 15-min access period and were roughly equivalent in caloric value.

\section{Method}

Subjects

Thirty naive, male Sprague Dawley rats weighing approximately $400 \mathrm{~g}$ at the start of testing were maintained as described in Experiment 1.

\section{Apparatus}

The solutions were prepared and presented as described in Experiment 1 . Glucose, maltose, and fructose were obtained from Sigma Chemical Co., St. Louis. Polycose was obtained from Ross Products Division, Abbott Laboratories, Columbus, $\mathrm{OH}$.

\section{Procedure}

The procedural details were identical to those of Experiment 1, except where noted. Separate groups of naive rats received $15-\mathrm{min}$ access to a given CS- $0.3 \mathrm{M}(5.41 \% \mathrm{w} / \mathrm{v})$ glucose $(n=8), 0.3 \mathrm{M}$ $(5.41 \% \mathrm{w} / \mathrm{v})$ fructose $(n=8), 0.1 \mathrm{M}(3.6 \% \mathrm{w} / \mathrm{v})$ maltose $(n=7)$, or $0.03 \mathrm{M}(3.0 \% \mathrm{w} / \mathrm{v})$ Polycose $(n=7)$. After a 15 -min interstimulus interval, all rats were injected i.p. with $0.15 \mathrm{M} \mathrm{LiCl}(1.33 \mathrm{ml} / 100 \mathrm{~g}$ b.w.). There were three such CS-US pairings, followed by a single 15-min test trial. Two water days (15-min access) elapsed between each. As mentioned in Experiment 1, the inclusion of animals in the extinction phase of the experiments required that the rats demonstrate robust CTA learning (i.e., a 75\% reduction in CS intake from Trial 1 to Trial 3). All animals in this experiment met this a priori requirement. Finally, all rats in this iteration were tested using the mixed extinction design described above. The data were analyzed as described in Experiment 1.

\section{Results}

\section{Acquisition}

As illustrated in Figure 4, the rats suppressed intake of all CSs following taste- $\mathrm{LiCl}$ pairings [main effect of trial: $F(3,78)=314.41, p<.0001]$. Rats in the fructose group consumed more fluid during Trial 1 than any other CS group [CS $\times$ trial interaction: $F(9,78)=3.06, p<$ .005 , with post hoc test]. Otherwise, intake did not differ across CS conditions ( $p$ s $>.05)$. Together, these data show that although fructose induced more intake on the first exposure, the development of the CTA was identical across CS conditions, with all groups acquiring a complete CTA at test $(p \mathrm{~s}<.05)$.

\section{Extinction}

Figure $5 \mathrm{~A}$ shows the mean $( \pm S E M)$ intake of water and CS solutions during the two-bottle tests. All rats received CS on the intervening one-bottle days. Figure 5B depicts the mean $( \pm S E M)$ 24-h CS intake during the one-bottle and two-bottle access periods. The one-bottle intake is the mean 24 -h intake during the $48 \mathrm{~h}$ of continuous access to the CS.

CS versus water intake (two-bottle). Glucose was replicated as a CS in Experiment 2, and the results of the two experiments were similar (compare Figures $3 \mathrm{Ad}$ and $5 \mathrm{Aa}$ ). This similarity demonstrates that the rapid extinction of glucose in Cycle 2 of Experiment 1 was not a fluke. In fact, this rapid extinction occurred not only for glucose, but also for maltose and Polycose, both glucose polymers. These relationships were confirmed by a threefactor ANOVA indicating that CS intake increased across two-bottle tests, while intake of water decreased $[F(4,104)=39.89, p<.0001]$. Given the similarities among the glucose-containing moieties, the three-factor interaction was nonsignificant $[F(12,104)=1.73, p>$ $.05]$. Consequently, separate two-factor ANOVAs were conducted for each CS varying stimulus (CS vs. water) and cycle (1-5). As in Experiment 1, the CTA for the glucose CS extinguished by the second two-bottle test $[F(4,28)=21.06, p<.01]$, such that intake of glucose did not differ from intake of water $(p>.05)$. Subsequent glucose intake was greater than intake of water during Cycles 3,4 , and $5(p \mathrm{~s}<.01)$. The two additional glucose polymers, maltose and Polycose, also elicited this rapid extinction. Post hoc analysis of the significant interaction for maltose $[F(4,24)=12.26, p<.05]$ and Polycose $[F(4,24)=5.83, p<.01]$ revealed that the CTA for these CSs also extinguished by the second two-bottle test ( $p$ s > .05). Like glucose, intake of maltose was greater than that of water during the third, fourth, and fifth cycles $(p s<.05)$. Except for the first cycle, intake of Polycose and water remained equivalent throughout testing $(p \mathrm{~s}>.05)$. In Experiment 2, only fructose exhibited the delayed extinction (Cycle 4) displayed by the other three CSs in Experiment 1, as revealed following post hoc analysis of the significant interaction $[F(4,28)=8.6$, $p<.01$ ] (i.e., the aversion to fructose, like those to sucrose and saccharin in Experiment 1, did not extinguish until the fourth cycle, $p>.05$ ).

CS intake (one-bottle vs. two-bottle test). As in Experiment 1 , the initial two-bottle CS intakes were well below one-bottle levels, irrespective of the CS moiety (Figure 5B). By Trial 2, however, two-bottle glucose, maltose, and Polycose intakes were not different than the preceding one-bottle day. In contrast, even though it increased across trials, two-bottle intake of fructose never approached the one-bottle volumes. The three-factor ANOVA revealed that CS intake was greater overall in the one-bottle than in the two-bottle test $[F(1,26)=71.9$, $p<.0001]$. The CS $\times$ bottle interaction was significant $[F(3,26)=3.57, p<.05]$, indicating that glucose and maltose intakes were the same regardless of the test ( $p$ s $>.05$ ), but the intakes of Polycose and fructose were greater when examined using the one-bottle test ( $p$ s $<$ .05 ). Once again, given the similar pattern of data across three of the four CSs, separate two-factor ANOVAs were conducted for each of the CSs varying bottle (one- vs. two-bottle) and cycle (1-5). For glucose, the two-factor interaction approached statistical significance $[F(4,56)=$ $2.45, p<.056]$, and post hoc tests on this interaction revealed that one-bottle intake was greater than two-bottle intake, but only during the first cycle $(p<.05)$. An identical pattern was evident for maltose [post hoc tests, bottle $\times$ trial interaction: $F(4,48)=3.47, p<.01]$. Intake of Polycose during the one- and two-bottle tests reflected a similar pattern over trials, but the interaction was not significant $[F(4,48)=1.21, p>.1]$. Finally, post hoc 


\section{A. CS vs. Water Intake (Two-Bottle)}

Aa. 0.3 M glucose

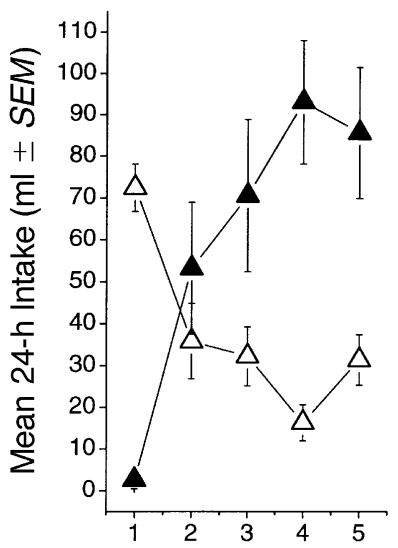

Ab. $0.1 \mathrm{M}$ maltose

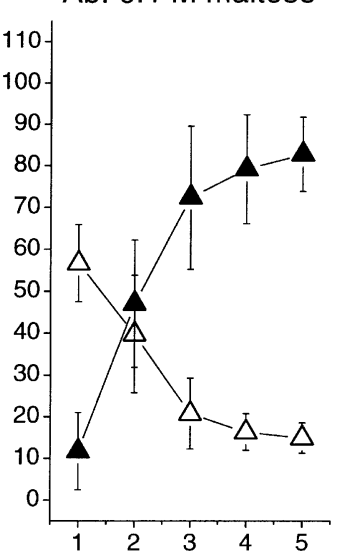

Ac. 0.03 M Polycose

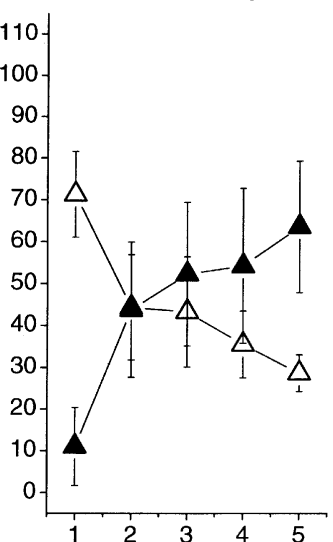

Ad. $0.3 \mathrm{M}$ fructose

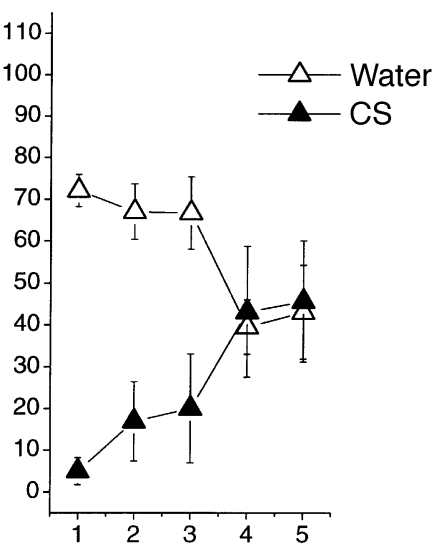

B. CS Intake One-Bottle vs. Two-Bottle

Ba. 0.3 M glucose

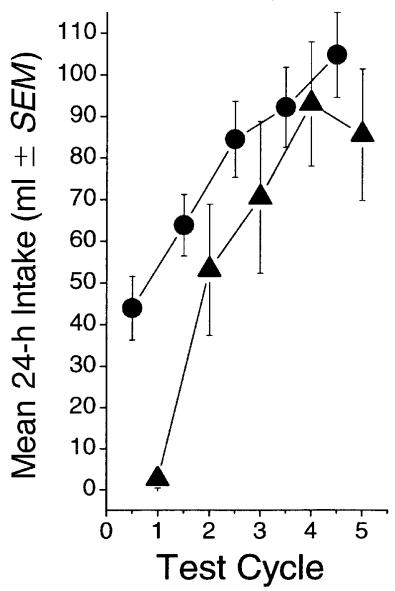

Bb. $0.1 \mathrm{M}$ maltose

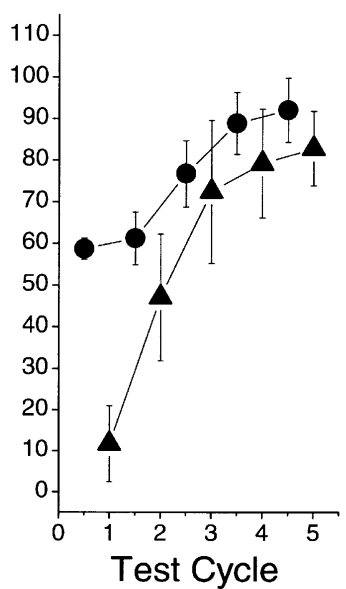

Bc. $0.03 \mathrm{M}$ Polycose

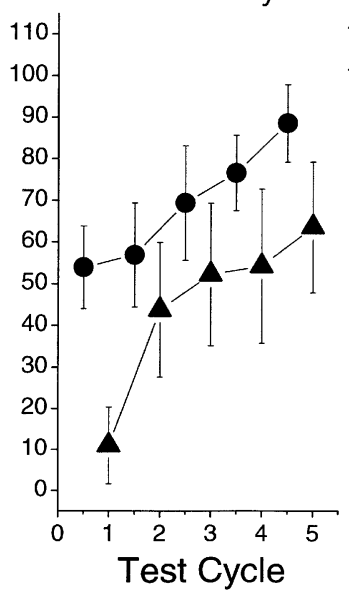

Bd. 0.3 M fructose

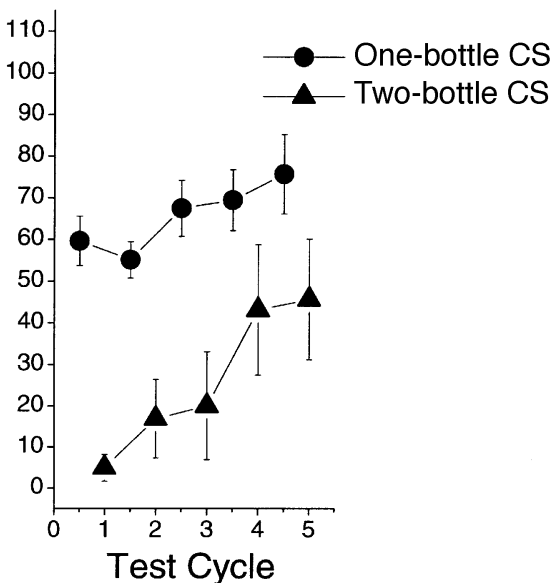

Figure 5. The top panels (A) show the mean ( \pm SEM) 24-h intake of water (open triangles) and CS solutions (filled triangles) during the two-bottle choice tests in Experiment 2. The bottom panels (B) show the mean ( $\pm S E M)$ 24-h CS intake during both the onebottle (filled circles) and two-bottle (filled triangles) access periods. The one-bottle intake is the mean $24-h$ intake during the 48 h of continuous access to the $\mathrm{CS}$.

tests of the significant bottle $\times$ trial interaction $[F(4,56)=$ $2.6, p<.05]$ revealed that rats ingested more fructose during the one-bottle than the two-bottle test across Cycles $1-5(p \mathrm{~s}<.05)$.

Taken together, the results of this experiment confirm that extinction of a CTA occurs rapidly when glucose serves as the gustatory CS. Moreover, the data show that a similar extinction pattern is evident for the three glucose moieties: glucose, maltose, and Polycose. Whereas rats in the Polycose condition never consumed significantly more of the CS than water, rats in the glucose and maltose groups evidenced a clear preference for the CS over water beginning with the third two-bottle test. Finally, the fructose aversion, like that to the saccharin, sucrose, and alanine CSs, is more resistant to extinction.

\section{GENERAL DISCUSSION}

The present study used a newly developed mixed extinction design that allowed for forced exposure to the CS during one-bottle access followed by a two-bottle probe test to evaluate the effect of the gustatory CS on the extinction of a CTA. Studies examining the extinction of a CTA are limited to relatively few taste stimuli, with the majority using saccharin (Bevins, Jensen, Hinze, \& Besheer, 1999; Grote \& Brown, 1971; McCaughey, Giza, Nolan, \& Scott, 1997; Nolan et al., 1997). In an attempt to understand whether CTA extinction is influenced by the nature of the gustatory cue, we expanded our investigation to include several carbohydrates, including monosaccharides (glucose, fructose), disaccharides (sucrose, maltose), a 
polysaccharide (Polycose), as well as saccharin and an amino acid (alanine).

The data revealed that the mixed extinction design is superior to either the one-bottle or the two-bottle test alone. This novel design combines the strengths of the individual procedures by facilitating CS intake during a one-bottle test and then probing for the expression of the CTA in a subsequent two-bottle test. The exclusive use of the two-bottle test was inadequate, because the animals failed to sample the CS significantly when presented in conjunction with a water alternative. Moreover, the sole use of the one-bottle test incorrectly indicated that the CTA had extinguished when, in fact, the CTA was simply obscured when thirsty rats were forced to consume the CS in the absence of an alternative source of fluid.

The use of the mixed extinction design also revealed that the rate of extinction of a CTA is dependent on the nature of the CS employed. Specifically, extinction of the CTA occurred by the second cycle when using a glucose, maltose, or Polycose CS, by the fourth cycle when using a saccharin, sucrose, or fructose CS, and by the sixth cycle when using an alanine CS. In the case of glucose and maltose, the CTA actually reversed to a preference. The question remains why a CTA to glucose, a glucose dimer, or a glucose polymer extinguished more quickly than a CTA to the other CSs. Differences in the rate of extinction could be attributed to a number of factors. These include the palatability/intensity of the CS, the strength of the initial CS-US association, or alternatively, to postingestive consequences of the CS.

Regarding the palatability or intensity of the CS, the selected gustatory stimuli do not appear to differ appreciably in either characteristic. With the exception of fructose, intakes of the various CSs during the first acquisition trial were nearly identical. Since the intake of $0.3 \mathrm{M}$ fructose on Trial 1 was greater than intake of the other CSs, the suggestion that intake of the other CSs was limited by a ceiling effect becomes less likely. Thus, although the CSs were not matched on intensity or palatability, as would be determined by a series of two-bottle preference tests, with the exception of fructose they were matched on intake (i.e., acceptance) in a one-bottle test.

Rate of extinction of a CTA can also be influenced by the strength of the initial conditioned response (Mikulka \& Klein, 1980). In Experiment 1, Trial 2, the magnitude of the CTA for saccharin was greater relative to sucrose or glucose. A possible explanation for this difference in the rate of acquisition of the CTA to saccharin may be the bitter component to saccharin (Dess, 1993), because CTA conditioning occurs more readily when aversive rather than preferred substances are utilized as the CS (Seligman, 1970). If the rate of extinction of the CTA in this experiment were linked to the strength of the CS-US association, however, we also would have expected the CTA to saccharin to have been more resistant to extinction. In fact, extinction of the CTA to saccharin was no slower than extinction of that to fructose or sucrose.
Moreover, although there were small differences in the rate of acquisition of the CTA, avoidance of CS at the time of the final test was nearly complete for all rats, regardless of the nature of the CS. Differences in extinction, therefore, do not appear to be linked to differences in the strength of the original CS-US association.

The final possibility that may account for the differences in the CTA extinction rates is the effect of postingestive feedback. This hypothesis suggests that extinction is augmented in the two-bottle test because of counterconditioning produced by the positive postingestive properties of the CS during the one-bottle access. This effect, termed conditioned flavor preference, is well established; it occurs whenever a distinctive oral stimulus is paired with an intragastric nutrient (see Azzara \& Sclafani, 1998). In fact, certain carbohydrates have been found to be more potent than others in producing conditioned flavor preferences. For example, intragastric infusions of $32 \%$ maltose are more reinforcing than intragastric infusions of $32 \%$ sucrose (Azzara \& Sclafani, 1998). Likewise, intragastric infusions of glucose yield stronger conditioned flavor preferences than do equimolar infusions of fructose (Ackroff, Touzani, Peets, \& Sclafani, 2001). These findings parallel the pattern of results obtained in the present report. That is, glucose and maltose condition stronger flavor preferences than sucrose and fructose do and, when used as CSs in a CTA, the former two extinguished more rapidly than did the latter. These data are consistent with the suggestion that glucose and other glucose moieties produce potent and rapid signals from both pre- and postabsorptive sites that are not generated by other sugars, including sucrose and fructose (Ackroff et al., 2001). These signals may support a conditioned flavor preference that counteracts the CTA. Taken together, these data suggest that not only acquisition (see Garcia, 1990), but also extinction of a CTA could be affected by the postingestive consequences of the gustatory stimulus.

The mechanisms by which forced consumption of certain glucose moieties override the negative association between the CS and the US more so than do other "sweet" tasting stimuli is presently unknown. One possible postingestive influence is the caloric content of the CS (Mehiel \& Bolles, 1984). For example, rats display a preference for the taste paired with the intragastric infusions of Polycose compared with a different taste paired with similar infusions of water or no infusion (Elizalde \& Sclafani, 1990; Sclafani \& Nissenbaum, 1988). Although nutritional value is a likely factor in the development of a conditioned taste preference, it is unlikely to account for the differential rates of extinction found here. In Experiment 1, the rate of extinction of the CTA to the nonnutritive sweetener saccharin was similar to that for sucrose (i.e., both occurred by the fourth $72-\mathrm{h}$ extinction cycle). Conversely, the concentrations selected for glucose and fructose were equicaloric, yet extinguished at completely different rates. These results are consistent with the findings that glucose has more 
potent postingestive properties than fructose (Sclafani \& Ackroff, 1994) and further challenge the notion that the caloric value of the CS, in and of itself, accounts for the differential rate of extinction of the CTAs. This possibility could be examined further by repeating the present experiments in sham-drinking rats, with or without concomitant intraduodenal nutritive infusions.

In sum, the present experiments demonstrate that a mixed extinction design serves as a more useful tool for assessing the extinction of a CTA than does either the one-bottle or the two-bottle test used alone. Using this paradigm, it became evident that the nature of the gustatory CS affected the rate of extinction of the LiCl-induced CTA. Specifically, a CTA to glucose or glucose moieties extinguished far more rapidly than did the CTA that was established to the other normally preferred chemicals. Indeed, for glucose and maltose, the CTA switched to a preference for the CS over water. Only equal preference for the CS and water was obtained with the other stimuli, despite repeated extinction cycles. The reason for this difference among the stimuli is not clear. The effect does not appear to be related to either the intensity or the palatability of the CS or to the strength of the initial CS-US association. Counterconditioning may account for the rapid extinction of the CTA to glucose, maltose, and Polycose, but neither the palatability nor the caloric value of the CS mediates this effect.

\section{REFERENCES}

Ackroff, K., Touzani, K., Peets, T. K., \& Sclafani, A. (2001). Flavor preferences conditioned by intragastric fructose and glucose: Differences in reinforcement potency. Physiology \& Behavior, 72, 691-703.

AzZara, A. V., \& Sclafani, A. (1998). Flavor preferences conditioned by intragastric sugar infusions in rats: Maltose is more reinforcing than sucrose. Physiology \& Behavior, 64, 535-541.

Berman, D. E., \& DudAI, Y. (2001). Memory extinction, learning anew, and learning the new: Dissociations in the molecular machinery of learning in cortex. Science, 291, 2417-2419.

Bevins, R. A., Jensen, H. C., Hinze, T. S., \& Besheer, J. (1999). Taste quality and extinction of a conditioned taste aversion in rats. Animal Learning \& Behavior, 27, 358-366.

DESS, N. K. (1993). Saccharin's aversive taste in rats: Evidence and implications. Neuroscience \& Biobehavioral Reviews, 17, 359-372.

Elizalde, G., \& SclafanI, A. (1990). Flavor preferences conditioned by intragastric polycose: A detailed analysis using the electronic esophagus preparation. Physiology \& Behavior, 47, 63-77.

Garcia, J. (1990). Learning without memory. Journal of Cognitive Neuroscience, 2, 287-305.

Garcia, J., \& Koelling, R. A. (1966). Relation of cue to consequence in avoidance learning. Psychonomic Science, 4, 123-124.
Grote, F. W., \& Brown, R. T. (1971). Conditioned taste aversions: Two-stimulus tests are more sensitive than one-stimulus tests. Behavior Research Methods \& Instrumentation, 3, 311-312.

Gutiérrez, H., Hernández-Echeagaray, E., Ramírez-Amaya, V. \& BermúdeZ-Rattoni, F. (1999). Blockade of $N$-methyl-D-aspartate receptors in the insular cortex disrupts taste aversion and spatial memory formation. Neuroscience, $\mathbf{8 9}, 751-758$.

Klosterhalfen, S., \& KlosterhalfeN, W. (1985). Conditioned taste aversion and traditional learning. Psychological Research, 47, 71-94.

McCaughey, S. A., Giza, B. K., Nolan, L. J., \& ScotT, T. R. (1997). Extinction of a conditioned taste aversion in rats: II. Neural effects in the nucleus of the solitary tract. Physiology \& Behavior, 61, 373-379.

MeHIEL, R., \& Bolles, R. C. (1984). Learned flavor preferences based on caloric outcome. Animal Learning \& Behavior, 12, 421-427.

MikulKa, P., \& Klein, S. (1980). Resistance to extinction of a taste aversion: Effects of level of training and procedures used in acquisition and extinction. American Journal of Psychology, 93, 631-641.

Nolan, L. J., McCaughey, S. A., Giza, B. K., RhineharT-Doty, J. A., Sмiтh, J. A., \& Scott, T. R. (1997). Extinction of a conditioned taste aversion in rats: I. Behavioral effects. Physiology \& Behavior, $\underline{\mathbf{6 1}}_{2}$ 319-323.

NORGREN, R., \& GRIGSON, P. S. (1996). The role of the central gustatory system in learned taste aversions. In T. Ono, B. McNaughton, S. Molotchnikoff, E. Rolls, \& H. Nishijo (Eds.), Perception, memory, and emotion: Frontiers in neuroscience (pp. 479-497). Tarrytown, NY: Pergamon.

ReILLY, S. (1998). The role of the gustatory thalamus in taste-guided behavior. Neuroscience \& Biobehavioral Reviews, 22, 883-901.

REILLY, S. (1999). The parabrachial nucleus and conditioned taste aversion. Brain Research Bulletin, 48, 239-254.

Revusky, S., \& GarCia, J. (1970). Learned associations over long delays. In G. H. Bower (Ed.), The psychology of learning and motivation (Vol. 4, pp. 1-84). New York: Academic Press.

SclafANi, A., \& ACKROFF, K. (1994). Glucose- and fructose-conditioned flavor preferences in rats: Taste versus postingestive conditioning. Physiology \& Behavior, 56, 399-405.

SCLAFANI, A., \& ClYNE, A. E. (1987). Hedonic response of rats to polysaccharide and sugar solutions. Neuroscience \& Biobehavioral Reviews, 11, 173-180.

ScLafani, A., \& Nissenbaum, J. W. (1988). Robust conditioned flavor preference produced by intragastric starch infusions in rats. American Journal of Physiology, 255, R672-R675.

Seligman, M. E. P. (1970). On the generality of the laws of learning. Psychological Review, 77, 406-418.

SPECTOR, A. C. (1995). Gustatory function in the parabrachial nuclei: Implications from lesion studies in rats. Review of Neuroscience, $\mathbf{6}$, 143-175.

Spector, A. C., Smith, J. C., \& Hollander, G. R. (1986). Radiationinduced taste aversion: Effect of radiation exposure level and the exposure-taste interval. Radiation Research, 106, 271-277.

TAYLOR, K. M., \& BOAKES, R. A. (2002). Extinction of a conditioned taste aversion: Effects of concentration and overshadowing. Quarterly Journal of Experimental Psychology, 55B, 213-239.

(Manuscript received July 4, 2002; revision accepted for publication June 21, 2004.) 